\title{
On the maximum number of distinct intersections in an intersecting family
}

\author{
Peter Frankl; , Sergei Kiselev ${ }^{\dagger}$ Andrey Kupavskii ${ }^{\ddagger}$
}

\begin{abstract}
For $n>2 k \geq 4$ we consider intersecting families $\mathcal{F}$ consisting of $k$-subsets of $\{1,2, \ldots, n\}$. Let $\mathcal{I}(\mathcal{F})$ denote the family of all distinct intersections $F \cap F^{\prime}, F \neq F^{\prime}$ and $F, F^{\prime} \in \mathcal{F}$. Let $\mathcal{A}$ consist of the $k$-sets $A$ satisfying $|A \cap\{1,2,3\}| \geq 2$. We prove that for $n \geq 50 k^{2}$ $|\mathcal{I}(\mathcal{F})|$ is maximized by $\mathcal{A}$.
\end{abstract}

\section{Introduction}

Let $n, k$ be positive integers, $n>2 k$. Let $X=\{1,2, \ldots, n\}$ be the standard $n$-element set and let $\left(\begin{array}{c}X \\ k\end{array}\right)$ be the collection of all its $k$-subsets. For a family $\mathcal{F} \subset\left(\begin{array}{l}X \\ k\end{array}\right)$ let $\mathcal{I}(\mathcal{F}):=\left\{F \cap F^{\prime}: F, F^{\prime} \in \mathcal{F}, F \neq F^{\prime}\right\}$ be the family of all distinct pairwise intersections. Recall that a family $\mathcal{F}$ is called intersecting if $F \cap F^{\prime} \neq \emptyset$ for all $F, F^{\prime} \in \mathcal{F}$.

One of the cornerstones of extremal set theory is the Erdös-Ko-Rado Theorem:

Theorem 1.1 ([EKR]). Suppose that $\mathcal{F} \subset\left(\begin{array}{l}X \\ k\end{array}\right)$ is intersecting. Then

$$
|\mathcal{F}| \leq\left(\begin{array}{l}
n-1 \\
k-1
\end{array}\right)
$$

*Rényi Institute, Budapest, Hungary and Moscow Institute of Physics and Technology, Russia, Email: peter.frankl@gmail.com

${ }^{\dagger}$ Moscow Institute of Physics and Technology, Email: kiselev.sg@gmail.com

${ }^{\ddagger}$ G-SCOP, CNRS, University Grenoble-Alpes, France and Moscow Institute of Physics and Technology, Russia; Email: kupavskii@yandex.ru. The authors acknowledge the financial support from the Russian Government in the framework of MegaGrant no 07515-2019-1926. 
For a fixed element $x \in X$ define the full star $\mathcal{S}_{x}$ by $\mathcal{S}_{x}:=\left\{S \in\left(\begin{array}{l}X \\ k\end{array}\right): x \in S\right\}$. Clearly $\mathcal{S}_{x}$ is intersecting and it provides equality in (1.1). Subfamilies of $\mathcal{S}_{x}$ are called stars. If we permit $n=2 k$ then there are many other intersecting families attaining equality in (1.1). However, Hilton and Milner [HM] proved that for $n>2 k$ the full stars are the only intersecting families with this property.

For a family $\mathcal{G} \subset 2^{X}$ define the family of transversals:

$$
\mathcal{T}(\mathcal{G}):=\{T \subset X:|T| \leq k, T \cap G \neq \emptyset \text { for all } G \in \mathcal{G}\} .
$$

With this definition $\mathcal{G} \subset\left(\begin{array}{l}X \\ k\end{array}\right)$ is intersecting iff $\mathcal{G} \subset \mathcal{T}(\mathcal{G})$. For $\mathcal{G} \subset 2^{X}$ and $0 \leq \ell \leq n$ define the $\ell$-th level of $\mathcal{G}$ by $\mathcal{G}^{(\ell)}:=\{G \in \mathcal{G},|G|=\ell\}$.

An intersecting family $\mathcal{F} \subset\left(\begin{array}{c}X \\ k\end{array}\right)$ is called saturated if $\mathcal{F} \cup\{G\}$ ceases to be intersecting for all $G \in\left(\begin{array}{l}X \\ k\end{array}\right) \backslash \mathcal{F}$.

Observation 1.2. An intersecting family $\mathcal{F} \subset\left(\begin{array}{l}X \\ k\end{array}\right)$ is saturated iff $\mathcal{F}=$ $\mathcal{T}(\mathcal{F})^{(k)}$.

The aim of the present paper is to investigate the maximum size of $\mathcal{I}(\mathcal{F})$ over intersecting families $\mathcal{F} \subset\left(\begin{array}{l}X \\ k\end{array}\right)$. Since $\mathcal{F} \subset \widetilde{\mathcal{F}}$ implies $\mathcal{I}(\mathcal{F}) \subset \mathcal{I}(\widetilde{\mathcal{F}})$, in the process we may assume that $\mathcal{F}$ is saturated.

Unless otherwise stated, all considered intersecting families are supposed to be saturated. We need the following lemma that was essentially proved in [F78]. In order to state it, recall that a family $\mathcal{B}$ is called an antichain if $B \not \subset B^{\prime}$ holds for all distinct members $B, B^{\prime} \in \mathcal{B}$. Recall also that an antichain $\left\{A_{1}, \ldots, A_{p}\right\}$ is called a sunflower of size $p$ with center $C$ if

$$
A_{i} \cap A_{j}=C \text { for all } 1 \leq i<j \leq p .
$$

Lemma 1.3. Suppose that $\mathcal{F} \subset\left(\begin{array}{l}X \\ k\end{array}\right)$ is a saturated intersecting family. Let $\mathcal{B}=\mathcal{B}(\mathcal{F})$ be the family of minimal (w.r.t. containment) sets in $\mathcal{T}(\mathcal{F})$. Then

(i) $\mathcal{B}$ is an intersecting antichain,

(ii) $\mathcal{F}=\left\{H \in\left(\begin{array}{l}X \\ k\end{array}\right): \exists B \in \mathcal{B}, B \subset H\right\}$,

(iii) $\mathcal{B}$ contains no sunflower of size $k+1$.

The proof is given in the next section.

Define the intersecting family $\mathcal{A}=\mathcal{A}(n, k)$ on the ground set $X=$ $\{1, \ldots, n\}$ by

$$
\mathcal{A}:=\left\{A \in\left(\begin{array}{c}
X \\
k
\end{array}\right):|A \cap\{1,2,3\}| \geq 2\right\} .
$$


The main result of the present paper is

Theorem 1.4. Suppose that $n \geq 50 k^{2}, k \geq 2$ and $\mathcal{F} \subset\left(\begin{array}{l}X \\ k\end{array}\right)$ is intersecting. Then

$$
|\mathcal{I}(\mathcal{F})| \leq|\mathcal{I}(\mathcal{A})|
$$

Let us note that it is somewhat surprising that the maximum is attained for $\mathcal{A}$ and not the full star which is much larger. Let us present the formula for $|\mathcal{I}(\mathcal{A})|$.

\section{Proposition 1.5.}

$$
|\mathcal{I}(\mathcal{A})|=3 \sum_{0 \leq i \leq k-2}\left(\begin{array}{c}
n-3 \\
i
\end{array}\right)+3 \sum_{0 \leq i \leq k-3}\left(\begin{array}{c}
n-3 \\
i
\end{array}\right)+\sum_{0 \leq i \leq k-4}\left(\begin{array}{c}
n-3 \\
i
\end{array}\right) .
$$

Proof. Let $A, A^{\prime} \in \mathcal{A}$. Then there are seven possibilities for $A \cap A^{\prime} \cap\{1,2,3\}$, namely, all non-empty subsets of $\{1,2,3\}$. If $A \cap A^{\prime} \cap\{1,2,3\}=\{1\}$ then $A \cap\{1,2,3\}$ and $A^{\prime} \cap\{1,2,3\}$ are $\{1,2\}$ and $\{1,3\}$ in some order. Since $n>2 k$ it is easy to see $A \cap A^{\prime}=\{1\} \cup D$ is possible for all $D \subset\{4, \ldots, n\}$, $|D| \leq k-2$.

The remaining six cases can be dealt similarly.

Note that the RHS of (1.3) can be simplified to

$$
3 \sum_{0 \leq i \leq k-2}\left(\begin{array}{c}
n-2 \\
i
\end{array}\right)+\sum_{0 \leq i \leq k-4}\left(\begin{array}{c}
n-3 \\
i
\end{array}\right) .
$$

In comparison

$$
\left|\mathcal{I}\left(\mathcal{S}_{x}\right)\right|=\sum_{0 \leq i \leq k-2}\left(\begin{array}{c}
n-1 \\
i
\end{array}\right)=2 \sum_{0 \leq i \leq k-2}\left(\begin{array}{c}
n-2 \\
i
\end{array}\right)-\left(\begin{array}{c}
n-2 \\
k-2
\end{array}\right) .
$$

That is,

$$
\left|\mathcal{I}\left(\mathcal{S}_{x}\right)\right|<\frac{2}{3}|\mathcal{I}(\mathcal{A})|
$$

Doing more careful calculations, one can replace $\frac{2}{3}$ with $\frac{n}{3(n-k)}$.

The paper is organized as follows. In Section 2 we prove Lemma 1.3 and the main lemma (Lemma 2.3) which provides some upper bounds concerning $\mathcal{B}(\mathcal{F})$. In Section 3 we prove Theorem 1.4. In Section 4 we mention some related problems. 


\section{Preliminaries and the main lemma}

Proof of Lemma 1.3. The fact that $\mathcal{B}$ is an antichain is obvious. Suppose for contradiction that $B, B^{\prime} \in \mathcal{B}$ but $B \cap B^{\prime}=\emptyset$. If $|B|=\left|B^{\prime}\right|=k$ then $B, B^{\prime} \in \mathcal{F}$ and $B \cap B^{\prime} \neq \emptyset$ follows. By symmetry suppose $\left|B^{\prime}\right|<k$. Now $\mathcal{B} \subset \mathcal{T}(\mathcal{F})$ implies that $B^{\prime} \cap F \neq \emptyset$ for all $F \in \mathcal{F}$. Choose a $k$-element superset $F^{\prime}$ of $B^{\prime}$ with $B \cap F^{\prime}=\emptyset$. Since $B^{\prime} \in \mathcal{T}(\mathcal{F})$, we have $F^{\prime} \in \mathcal{T}(\mathcal{F})$. By Observation 1.2, $F^{\prime} \in \mathcal{F}$. However, $B \cap F^{\prime}=\emptyset$ contradicts $B \in \mathcal{T}(\mathcal{F})$. This proves (i). Statement (ii) is immediate from the definition of $\mathcal{B}$.

To prove (iii) suppose for contradiction that $B_{0}, B_{1}, \ldots, B_{k} \in \mathcal{B}$ form a sunflower with center $C$. Since $\mathcal{B}$ is an antichain, $C$ is a proper subset of $B_{0}$. Consequently $C \notin \mathcal{T}(\mathcal{F})$. Thus we may choose $F \in \mathcal{F}$ satisfying $C \cap F=\emptyset$.

Consider the $k+1$ pairwise disjoint sets $B_{0} \backslash C, \ldots, B_{k} \backslash C$. By $|F|=k$ there is some $i, 0 \leq i \leq k$ with $F \cap B_{i} \backslash C=\emptyset$. However this implies $F \cap B_{i}=\emptyset$, a contradiction.

In what follows, $\mathcal{B}:=\mathcal{B}(\mathcal{F})$ is as in Lemma 1.3: the family of minimal transversals of $\mathcal{F}$. Let us recall the Erdős-Rado Sunflower Lemma.

Lemma 2.1 ([ER]). Let $\ell \geq 1$ be an integer and $\mathcal{D} \subset\left(\begin{array}{c}X \\ \ell\end{array}\right)$ a family which contains no sunflower of size $k+1$. Then

$$
|\mathcal{D}| \leq \ell ! k^{\ell} .
$$

The following statement is both well-known and easy.

Lemma 2.2. Suppose that $\mathcal{E} \subset\left(\begin{array}{l}X \\ 2\end{array}\right)$ is intersecting, then either $\mathcal{E}$ is a star or a triangle.

We are going to use the standard notation: for integers $a \leq b$ we set $[a, b]=\{i: a \leq i \leq b\}$ and $[n]=[1, n]$. We also write $(x, y)$ instead of $\{x, y\}$ if $x \neq y$.

Based on Lemma 2.1 we could prove (1.2) for $n>k+50 k^{3}$. To get a quadratic bound we need to improve it under our circumstances. To state our main lemma we need some more definitions.

Define $t=t(\mathcal{B}):=\min \{|B|: B \in \mathcal{B}\}$. The covering number $\tau(\mathcal{B})$ is defined as follows: $\tau(\mathcal{B}):=\min \{|T|: T \cap B \neq \emptyset$ for all $B \in \mathcal{B}\}$. Since $\mathcal{F}$ is a saturated intersecting family, using (ii) of Lemma 1.3 we have $\tau(\mathcal{B})=t$.

Now we can present our main lemma that is a sharpening of a similar result in [F17]. Put $\mathcal{B}^{(\leq \ell)}:=\bigcup_{i=1}^{\ell} \mathcal{B}^{(\ell)}$. 
Lemma 2.3. Let $\ell$ be an integer, $k \geq \ell \geq 2$. Suppose that $\mathcal{F} \subset\left(\begin{array}{l}X \\ k\end{array}\right)$ is a saturated intersecting family, $\mathcal{B}=\mathcal{B}(\mathcal{F}), t \geq 2$. Assume that $\tau\left(\mathcal{B}^{(\leq \ell)}\right) \geq 2$. Then

$$
\left|\mathcal{B}^{(\ell)}\right| \leq t \cdot \ell \cdot k^{\ell-2}
$$

Proof. For the proof we use a branching process. We need some notation. During the proof a sequence is an ordered sequence of distinct elements of $X$ : $\left(x_{1}, x_{2}, \ldots, x_{s}\right)$. Sequences are denoted by $S, S_{1}$ etc. and we let $\widehat{S}$ denote the underlying unordered set: $\widehat{S}=\left\{x_{1}, x_{2}, \ldots, x_{s}\right\}$.

To start the branching process, we fix a set $B_{1} \in \mathcal{B}$ with $\left|B_{1}\right|=t(\mathcal{B})$ and for each element $y_{1} \in B_{1}$ we assign weight $t(\mathcal{B})^{-1}$ to the sequence $\left(y_{1}\right)$.

At the first stage, we replace each 1 -sequence $\left(y_{1}\right)$ with at most $\ell 2$ sequences: using $\tau\left(\mathcal{B}^{(\leq \ell)}\right) \geq 2$ we choose an arbitrary $B\left(y_{1}\right) \in \mathcal{B}$ satisfying $y_{1} \notin B\left(y_{1}\right),\left|B\left(y_{1}\right)\right| \leq \ell$, and assign weight $\left(t(\mathcal{B}) \cdot\left|B\left(y_{1}\right)\right|\right)^{-1} \geq(t(\mathcal{B}) \cdot \ell)^{-1}$ to each sequence $\left(y_{1}, y_{2}\right), y_{2} \in B\left(y_{1}\right)$. Note that the total weight assigned is exactly 1.

At each subsequent stage we pick a sequence $S=\left(x_{1}, \ldots, x_{p}\right)$ with weight $w(S)$ such that there exists $B \in \mathcal{B}$ satisfying $\widehat{S} \cap B=\emptyset$. Then we replace $S$ by the $|B|$ sequences $\left(x_{1}, \ldots, x_{p}, y\right), y \in B$, and assign weight $\frac{w(S)}{|B|}$ to each of them.

We continue until $\widehat{S} \cap B \neq \emptyset$ holds for all sequences $S$ and all $B \in \mathcal{B}$. Since $X$ is finite, this eventually happens. Importantly, the total weight assigned is still 1 .

Claim 2.4. For each $B \in \mathcal{B}^{(\ell)}$ there is some sequence $S$ with $\widehat{S}=B$.

Proof. Let us suppose the contrary. Since $\mathcal{B}$ is intersecting, a sequence with $\widehat{S}=B$ is not getting replaced by a longer sequence during the process. Let $S=\left(x_{1}, \ldots, x_{p}\right)$ be a sequence of maximal length that occurred at some stage of the branching process satisfying $\widehat{S} \varsubsetneqq B$. Since $\widehat{S}$ is a proper subset of $B, \widehat{S} \cap B^{\prime}=\emptyset$ for some $B^{\prime} \in \mathcal{B}$. Thus at some point we picked $S$ and chose some $\widetilde{B} \in \mathcal{B}$ disjoint to it. Since $\mathcal{B}$ is intersecting, $B \cap \widetilde{B} \neq \emptyset$. Consequently, for each $y \in B \cap \widetilde{B}$ the sequence $\left(x_{1}, x_{2}, \ldots, x_{p}, y\right)$ occurred in the branching process. This contradicts the maximality of $p$.

Let us check the weight assigned to $S$ with $|\widehat{S}|=\ell$. It is at least $1 /$ $\left(t(\mathcal{B}) \ell k^{\ell-2}\right)$. Since the total weight is $1,(2.2)$ follows. 
We should remark that the same $B \in \mathcal{B}^{(\ell)}$ might occur as $\widehat{S}$ for several sequences $S$ and for many sequences $|\widehat{S}| \neq \ell$ might hold. This shows that there might be room for considerable improvement.

Let us mention that if $\mathcal{F} \subset\left(\begin{array}{l}X \\ k\end{array}\right)$ is a saturated intersecting family with $\tau(\mathcal{F})=k$ then $\mathcal{B}(\mathcal{F})=\mathcal{F}$ and (2.2) reduces to $|\mathcal{F}| \leq k^{k}$, an important classical result of Erdős and Lovász [EL].

\section{The proof of Theorem 1.4}

Since the case $k=2$ trivially follows from Lemma 2.2 , we assume $k \geq 3$. Take any saturated intersecting $\mathcal{F}$ and let $\mathcal{B}=\mathcal{B}(\mathcal{F})$. First recall that for the full star $\mathcal{S}_{x}$

$$
\left|\mathcal{I}\left(\mathcal{S}_{x}\right)\right|=\sum_{0 \leq \ell \leq k-2}\left(\begin{array}{c}
n-1 \\
\ell
\end{array}\right)
$$

which is less than $|\mathcal{I}(\mathcal{A})|$, and so we may assume that $\mathcal{F}$ is not the full star, i.e., $\mathcal{B}^{(1)}=\emptyset$.

Recall that $t=\min \{|B|: B \in \mathcal{B}\}$. Let us first present two simple inequalities for sums of binomial coefficients that we need in the sequel.

$$
\left(\begin{array}{c}
n-a \\
i
\end{array}\right) /\left(\begin{array}{c}
n-a \\
i-1
\end{array}\right)=\frac{n-a-i+1}{i} \geq \frac{n-k}{k-1}
$$

for $k>i>0, a \geq 0$ and $i+a \leq k+1$. Thus for every $1 \leq s \leq k-1$

$$
\begin{gathered}
\sum_{0 \leq i \leq s}\left(\begin{array}{c}
n-2 \\
i
\end{array}\right) \geq \frac{n-k}{k-1} \sum_{0 \leq i \leq s-1}\left(\begin{array}{c}
n-2 \\
i
\end{array}\right) . \\
\left(\begin{array}{c}
n-2 \\
i
\end{array}\right) /\left(\begin{array}{c}
n \\
i
\end{array}\right)=\frac{(n-i)(n-i-1)}{n(n-1)}>\left(1-\frac{k}{n}\right)^{2} \text { for } 1<i<k .
\end{gathered}
$$

Thus

$$
\sum_{0 \leq i \leq s}\left(\begin{array}{c}
n-2 \\
i
\end{array}\right) \geq\left(1-\frac{k}{n}\right)^{2} \sum_{0 \leq i \leq s}\left(\begin{array}{l}
n \\
i
\end{array}\right) \text { for } 1<s<k
$$

Let us partition $\mathcal{F}$ into $\mathcal{F}^{(t)} \cup \ldots \cup \mathcal{F}^{(k)}$ where $F \in \mathcal{F}^{(\ell)}$ if $\ell=\max \{|B|: B \in$ $\mathcal{B}, B \subset F\}$. 
Set $\mathcal{I}_{\ell}=\left\{F \cap F^{\prime}: F \in \mathcal{F}^{(\ell)}, F^{\prime} \in \mathcal{F}^{(t)} \cup \ldots \cup \mathcal{F}^{(\ell)}\right\}$. In human language, if $F \in \mathcal{F}^{(\ell)}, F^{\prime} \in \mathcal{F}^{\left(\ell^{\prime}\right)}$ then we put $F \cap F^{\prime}$ into $\mathcal{I}_{\ell}$ iff $\ell^{\prime} \leq \ell$. It should be clear that

$$
|\mathcal{I}(\mathcal{F})| \leq \sum_{t \leq \ell \leq k}\left|\mathcal{I}_{\ell}\right|
$$

The point is that for $F \in \mathcal{F}^{(\ell)}$ and $B \subset F, B \in \mathcal{B}^{(\ell)}$, for an arbitrary $F^{\prime} \in \mathcal{F}$, $F \cap F^{\prime}$ is partitioned as

$$
F \cap F^{\prime}=\left(B \cap F^{\prime}\right) \cup\left((F \backslash B) \cap F^{\prime}\right) .
$$

Here there are at most $2^{\ell}-1$ possibilities for $B \cap F^{\prime}$ and $(F \backslash B) \cap F^{\prime}$ is a subset of $X$ of size at most $k-\ell$. This proves

Lemma 3.1. For any $t \leq \ell \leq k$ such that $\tau\left(\mathcal{B}^{(\leq \ell)}\right) \geq 2$ we have

$$
\left|\mathcal{I}_{\ell}\right| \leq\left(2^{\ell}-1\right)\left|\mathcal{B}^{(\ell)}\right| \sum_{0 \leq i \leq k-\ell}\left(\begin{array}{c}
n \\
i
\end{array}\right)<2^{\ell} \cdot \ell^{2} k^{\ell-2} \sum_{0 \leq i \leq k-\ell}\left(\begin{array}{c}
n \\
i
\end{array}\right)=: f(n, k, \ell)
$$

Note that if $\tau\left(\mathcal{B}^{(2)}\right)=2$ then $\mathcal{F}$ coincides with $\mathcal{A}$ and we have nothing to prove. Let $\alpha$ be the smallest integer such that $\tau\left(\mathcal{B}^{(\leq \alpha)}\right) \geq 2$. We have $\alpha \geq 3$. The family $\mathcal{F}^{\prime}:=\bigcup_{i=1}^{\alpha-1} \mathcal{F}^{(i)}$ is a trivial intersecting family, and thus

$$
\left|\bigcup_{i=1}^{\alpha-1} \mathcal{I}_{i}\right| \leq\left|\mathcal{I}\left(\mathcal{S}_{x}\right)\right| .
$$

On the other hand, using (3.2) it is clear that for $\ell \geq 2$

$$
f(n, k, \ell) / f(n, k, \ell+1)>\frac{(n-k) \ell^{2}}{2 k^{2}(\ell+1)^{2}} \geq 6 \text { for } n \geq 50 k^{2} .
$$

Hence

$$
\sum_{\ell=\alpha}^{k}\left|\mathcal{I}_{\ell}\right|<\sum_{3 \leq \ell \leq k} f(n, k, \ell)<\frac{6}{5} f(n, k, 3) .
$$

Summing the right hand sides of (3.5) and (3.6), we get that

$$
|\mathcal{I}(\mathcal{F})| \leq \sum_{0 \leq i \leq k-2}\left(\begin{array}{c}
n-1 \\
i
\end{array}\right)+\frac{432 k}{5} \sum_{0 \leq i \leq k-3}\left(\begin{array}{c}
n \\
i
\end{array}\right)
$$




$$
\begin{aligned}
& <\left(\begin{array}{l}
n-2 \\
k-2
\end{array}\right)+\left(\frac{432 k}{5}+2\right) \sum_{0 \leq i \leq k-3}\left(\begin{array}{l}
n \\
i
\end{array}\right) \\
& \stackrel{(3.2)}{\leq}\left(\begin{array}{l}
n-2 \\
k-2
\end{array}\right)+90 \frac{k(k-1)}{n-k} \sum_{0 \leq i \leq k-2}\left(\begin{array}{l}
n \\
i
\end{array}\right) \leq\left(\begin{array}{l}
n-2 \\
k-2
\end{array}\right)+1.8 \sum_{0 \leq i \leq k-2}\left(\begin{array}{c}
n \\
i
\end{array}\right) \\
& \stackrel{(3.3)}{<}\left(\begin{array}{l}
n-2 \\
k-2
\end{array}\right)+2 \sum_{0 \leq i \leq k-2}\left(\begin{array}{c}
n-2 \\
i
\end{array}\right)<|\mathcal{I}(\mathcal{A})| .
\end{aligned}
$$

\section{Concluding remarks}

Let $n_{0}(k)$ be the smallest integer such that Theorem 1.4 is true for $n \geq n_{0}(k)$. We proved $n_{0}(k) \leq 50 k^{2}$. One can improve on the constant 50 by being more careful in the analysis. The following example shows that $n_{0}(k) \geq(3-\varepsilon) k$.

For $1 \leq p \leq k, n>2 k$, define the family $\mathcal{B}_{p}(n, k)$ by

$$
\mathcal{B}_{p}(n, k):=\left\{A \in\left(\begin{array}{c}
{[n]} \\
k
\end{array}\right):|A \cap[2 p-1]| \geq p\right\} .
$$

Note that $\mathcal{S}_{1}=\mathcal{B}_{1}(n, k)$ and $\mathcal{A}=\mathcal{B}_{2}(n, k)$. It is easy to verify that

$$
\begin{aligned}
\left|\mathcal{I}\left(\mathcal{B}_{p}(n, k)\right)\right| & =\sum_{i=1}^{p-1}\left(\begin{array}{c}
2 p-1 \\
i
\end{array}\right) \sum_{j=0}^{k-p}\left(\begin{array}{c}
n-2 p+1 \\
j
\end{array}\right) \\
& +\sum_{i=p}^{2 p-1}\left(\begin{array}{c}
2 p-1 \\
i
\end{array}\right) \sum_{j=0}^{k-i-1}\left(\begin{array}{c}
n-2 p+1 \\
j
\end{array}\right) .
\end{aligned}
$$

By doing some calculations, one can see that $\left|\mathcal{I}\left(\mathcal{B}_{3}(n, k)\right)\right|>\left|\mathcal{I}\left(\mathcal{B}_{2}(n, k)\right)\right|$ for $n<(3-\varepsilon) k$. It would be interesting do decide, whether for $n>(1+\varepsilon) k$ the maximum is always attained on one of the families $\mathcal{B}_{p}(n, k)$.

Note that for $n=2 k, k \geq 14$, it is possible to construct an intersecting family $\mathcal{F}$ with $|\mathcal{I}(\mathcal{F})|=\sum_{i=0}^{k-1}\left(\begin{array}{c}n \\ i\end{array}\right)$ using an argument from [FKKP]. We say that a family $\mathcal{F}$ almost shatters a set $X \subset[n]$ if for any $A \subset X, A \notin\{\emptyset, X\}$, there is $F \in \mathcal{F}$ such that $F \cap X=A$. Take a random intersecting family $\mathcal{F}$ by picking a $k$-set from each pair $(A,[n] \backslash A)$ independently at random. In [FKKP, Theorem 7] it is proved, that with positive probability $\mathcal{F}$ almost shatters every $X \in\left(\begin{array}{c}{[2 k]} \\ k\end{array}\right)$. Fix such a family $\mathcal{F}$; then, by applying the almost shattering property two times, it is easy to show that, for each $I \subset[n]$, $1 \leq|I|<k$, there are two sets $F_{1}, F_{2} \in \mathcal{F}$, such that $I \subset F_{1}$ and $F_{1} \cap F_{2}=I$. 
Another natural problem is to consider $\widetilde{\mathcal{I}}(\mathcal{F})=\left\{F \cap F^{\prime}: F, F^{\prime} \in \mathcal{F}\right\}=$ $\mathcal{I}(\mathcal{F}) \cup \mathcal{F}$. Essentially the same proof shows that $\widetilde{\mathcal{I}}(\mathcal{F})$ is maximised by $\mathcal{F}=\mathcal{S}_{x}$ for $n \geq 50 k^{2}$ and one can verify that $\left|\mathcal{I}\left(\mathcal{B}_{2}(n, k)\right)\right|>\left|\mathcal{I}\left(\mathcal{S}_{x}\right)\right|$ for $n<(5-\varepsilon) k$.

In [F20] the analogous problem for the number of distinct differences $F \backslash F^{\prime}$ was considered. Improving those results in [FKK], we proved that for $n>50 k \cdot \log k$ the maximum is attained for the full star, $\mathcal{S}_{x}$. We showed also that it is no longer true for $n=c k, 2 \leq c<4, k>k_{0}(c)$.

The methods used in [FKK] are completely different.

\section{References}

[EKR] P. Erdős, C. Ko and R. Rado, Intersection theorems for systems of finite sets, The Quart. J. Math. Oxford, Ser. (2) 12 (1961), 313-320.

[EL] P. Erdős and L. Lovász, Problems and results on 3-chromatic hypergraphs and some related questions. In: Infinite and Finite Sets (Proc. Colloq. Math. Soc. J. Bolyai 10), edited by A. Hajnal et al., North-Holland, Amsterdam, 1975, 609-627.

[ER] P. Erdős and R. Rado, Intersection theorems for systems of sets, J. London Math. Soc. 35 (1960), 85-90.

[F78] P. Frankl, On intersecting families of finite sets, J. Combinatorial Theory A 24 (1978), 146-161.

[F17] P. Frankl, Antichains of fixed diameter, Moscow J. Combinatorics and Number Theory 7 (2017), 189-219.

[F20] P. Frankl, On the number of distinct differences in an intersecting family, Discrete Mathematics 344.2 (2021): 112210.

[FKK] P. Frankl, S. Kiselev and A. Kupavskii, Best possible bounds on the number of distinct differences in intersecting families, arXiv preprint arXiv:2106.05355 (2021).

[FKKP] N. Frankl, S. Kiselev, A. Kupavskii and B. Patkós, VC-saturated set systems, arXiv preprint arXiv:2005.12545 (2020).

[HM] A. J. W. Hilton and E. C. Milner, Some intersection theorems for systems of finite sets, Quart. J. Math. Oxford (2) 18 (1967), 369-384. 